\title{
Inequidad de la atención de las cardiopatías congénitas en los hospitales públicos de México. El falso derecho a la salud
}

\section{Inequity of congenital heart disease care in the public hospitals of Mexico. The false right to health}

\author{
Rocío A. Peña-Juárez *, Miguel A. Medina-Andrade y María T. Martínez-González \\ ${ }^{1}$ Servicio de Cardiología Pediátrica, Hospital General de Occidente; ${ }^{2}$ Servicio de Cirugía Cardiovascular Pediátrica, Hospital Civil Fray Antonio \\ Alcalde; ${ }^{3}$ Servicio de Pediatría, Hospital General de Occidente. Zapopan, Jalisco, México
}

\begin{abstract}
Resumen
La cardiología pediátrica y la cirugía cardiovascular han tenido avances importantes en los últimos años; las cardiopatías congénitas (CC) son una de las principales causas de mortalidad en niños. Muchos de los factores que determinan la evolución final de estos pacientes incluyen el tipo de cardiopatía y el tiempo del diagnóstico y tratamiento; infortunadamente, dos de los que presentan mayores efectos son el estado socioeconómico y el área geográfica de atención en México. El objetivo de este estudio es conocer el tipo de atención para los pacientes con CC en hospitales públicos del país.
\end{abstract}

Palabras clave: Cardiopatía congénita. Cardiología pediátrica. Cirugía.

\begin{abstract}
Pediatric cardiology and cardiovascular surgery have made significant advances in recent years, congenital heart diseases $(C H D)$ are one of the leading causes of mortality in children. Many of the factors that determine the final evolution of these patients include the type of heart disease, the time of diagnosis and treatment; unfortunately, in our country, two of those greatest impact area the socioeconomic status and the geographic area of attention. The objective of this study is to know the type of care for patients with CHD in public hospitals in the country.
\end{abstract}

Key words: Congenital heart disease. Pediatric cardiology. Surgery.

Los avances en cardiología pediátrica han sido notables en sumo grado y para entender el contexto es necesario describir una breve perspectiva histórica de la especialidad. En las primeras décadas del siglo pasado, el estudio de las cardiopatías congénitas (CC) tenía tan sólo un interés académico; John Lovett escribió en 1926: "Afortunadamente, el diagnóstico de la lesión exacta no es de gran importancia (...) no hay tratamiento curativo ni cosa alguna que disminuya las deformidades o favorezca el cierre de las aberturas anormales. Por tanto, el tratamiento debe ser higiénico y sintomático"1.

\section{Correspondencia:}

*Rocío A. Peña-Juárez

E-mail: alepejz@gmail.com
Fecha de recepción: 04-02-2020

Fecha de aceptación: 25-03-2020

DOI: 10.24875/ACM.20000034
Disponible en internet: $14-09-2020$ Arch Cardiol Mex. 2020;90(3):336-340 www.archivoscardiologia.com 1405-9940 / @ 2020 Instituto Nacional de Cardiología Ignacio Chávez. Publicado por Permanyer. Este es un artículo open access bajo la licencia CC BY-NC-ND (http://creativecommons.org/licenses/by-nc-nd/4.0/). 
En 1936 apareció el primer tratado sobre CC que publicó M. Abbott (Atlas of Congenital Heart Disease) y con posterioridad, en 1947, Helen B. Taussing publicó su libro Congenital Malformation of the Heart ${ }^{1,2}$; estos manuales, junto con las primeras intervenciones quirúrgicas de Robert E. Gross en $1938^{3}$, quien realizó con éxito la primera ligadura de un conducto arterioso permeable, dieron inicio al tratamiento quirúrgico de las $\mathrm{CC}^{2}$. El extraordinario desarrollo actual del conocimiento y el tratamiento de las $\mathrm{CC}$ fue posible debido a cinco grandes factores: la cirugía cardíaca, el cateterismo, la aparición de las prostaglandinas $\mathrm{E}_{1}$, el ecocardiograma 2D Doppler y la cardiología intervencionista. Sin duda alguna, la aplicación de la bomba de circulación extracorpórea a la cirugía cardíaca y la técnica de hipotermia profunda con paro circulatorio total hicieron posible el tratamiento quirúrgico a corazón abierto de casi todas las malformaciones cardíacas, incluso en el periodo neonatal ${ }^{2}$. En la actualidad, los avances en la cardiología pediátrica no sólo se limitan a progresos diagnósticos (ecocardiograma 4D, técnicas para evaluar la contractilidad ventricular como la deformación miocárdica) e imagen cardiovascular en la que puede realizarse una impresión en 3D antes de los procedimientos quirúrgicos, sino además a innumerables avances en electrofisiología cardíaca, cirugía cardiovascular, intervencionismo, perfusión, anestesia cardiovascular, cuidados posquirúrgicos cardiovasculares (tanto médicos como de enfermería), ecocardiograma e intervencionismo fetal; $y$, en fecha más reciente, se han observado avances en la rehabilitación cardíaca pediátrica. Hoy en día, esta fase de desarrollo y expansión de la especialidad se halla en su culmen, al atender de una forma integral al niño con CC o adquirida, y abarca desde el diagnóstico y el tratamiento prenatal de las $\mathrm{CC}$ hasta el seguimiento de los adolescentes supervivientes. Más aún, la mejoría del pronóstico de muchos de estos enfermos ha creado una nueva subespecialidad enfocada en las CC en el adulto ${ }^{4}$. Todos estos avances se llevan a cabo en muchos de los institutos de salud, aunque por desgracia este nivel de atención para las CC en México no se proporciona de forma equitativa.

Según el último estudio global, las CC causaron 261,247 muertes en el 2017, lo que representa una disminución de $34.7 \%$ en comparación con 1990, con 180,624 muertes en menores de un año. Los intervalos de mortalidad de CC decrecen con el aumento de los índices sociodemográficos; en consecuencia, muchas de estas muertes ocurrieron en países con ingresos bajos o medios. La prevalencia de las CC se ha elevado $18.7 \%$ entre 1990 y 2017; es decir, 11,998,283 personas viven con $\mathrm{CC}^{5}$, lo que indica una mejoría en la supervivencia y desde luego un mejor diagnóstico.

Las CC representan la forma más frecuente de defectos graves al nacimiento. En forma general, se considera que de los 130 millones de nacimientos al año en todo el mundo, más de un millón lo hace con alguna CC, lo que constituye un problema de salud pública mundial ${ }^{6}$. La incidencia de $\mathrm{CC}$ graves se aproxima a 2.5 a 3 por cada 1,000 nacimientos. En el año 2000 se calculaba una prevalencia global de 11.9 por cada 1,000 niños. En México se desconoce la prevalencia real de las CC; sin embargo, la información disponible se basa en las tasas de mortalidad del 2015. Las CC corresponden a la segunda causa de mortalidad en los menores de un año y, en el intervalo de 1 a 14 años, las CC pasaron de la tercera a la segunda causa al desplazar al cáncer infantil ${ }^{6,7}$. De acuerdo con la propuesta de regionalización de Calderón, et al., en el 2010 se requerían alrededor de 25 centros de cirugía cardíaca en México, pero sólo existen 10 centros médico-quirúrgicos especializados en la atención de estos pacientes, ocho de los cuales se encuentran en la Ciudad de México, uno en Monterrey y otro en Guadalajara; este último pertenece al Instituto Mexicano del Seguro Social, por lo que sólo atiende a la población asegurada. La propuesta de regionalización tenía como objetivo inicial fortalecer a 11 centros hospitalarios en distintas regiones del país para alcanzar un número mínimo de 21 sedes y resolver las cardiopatías más comunes (conducto arterioso, comunicación interauricular y comunicación interventricular) en los hospitales estatales o centros de alta especialidad cercanos al lugar de residencia del paciente, de tal modo que se redujera el costo familiar, social y la carga asistencial en los hospitales de referencia. Además, esta propuesta indicaba que si se añaden a las tres cardiopatías ya mencionadas el tratamiento de la coartación aórtica no compleja, la fístula sistémico-pulmonar, la tetralogía de Fallot y la atresia pulmonar con comunicación interventricular con ramas confluentes, toda vez que son muy frecuentes, su atención permitiría alcanzar entre 70 y $75 \%$ del total de las anomalías congénitas cardíacas y del porcentaje restante se harían cargo los 10 centros especializados disponibles en la actualidad. En una siguiente etapa, en los centros regionales de especialidad para la atención de los niños con CC, se practicarían de manera paulatina operaciones cardiovasculares neonatales de mayor complejidad y de esa 
manera sólo se derivaría a los pacientes con malformaciones cardíacas complejas ${ }^{7}$. Sin embargo, a 10 años de esta propuesta de regionalización, ¿cuánto se ha avanzado? Infortunadamente, esta propuesta aún está lejos de cumplirse.

En la cardiología pediátrica se requiere un alto grado de especialización y tecnificación y es la base que permite obtener resultados óptimos de supervivencia y calidad de vida en esta población. Esto significa que se requiere un equipo multidisciplinario tanto médico como de recursos para la atención integral de estos pacientes $y$, aunque los hospitales regionales cuenten con excelentes recursos humanos y se formen equipos multidisciplinarios, en muchas ocasiones no se dispone de los recursos materiales para instituir tratamientos de forma oportuna y calidad; es por ello que las cifras entre los institutos de salud y los hospitales estatales divergen en grado notorio.

En un plano regional existen hospitales cuya mortalidad quirúrgica es de $20 \%$ en cardiopatías con RACH 1 a 3 y otros en los que, al no contar con los recursos materiales para ofrecer algún tipo de tratamiento, registran tasas de mortalidad en cardiopatías críticas hasta de $80 \% 6$; además, puesto que no se tiene el material para su atención, en los estados no se modifica la mortalidad de las CC ni es posible ofrecer la mínima cobertura necesaria para la población que lo requiere; en consecuencia, se continúa la centralización de los servicios de cardiología pediátrica y ello da lugar al elevado número de pacientes en lista de espera en los institutos de salud.

Ante estos hechos, muchos grupos cardiológicos, en virtud del evidente escaso progreso para la atención de las CC en México, han decidido crear fundaciones cuyo objetivo es ofrecer atención oportuna y de calidad a estos pacientes, a través de recursos privados, públicos o ambos. Algunas de estas fundaciones celebran jornadas de cirugía cardiovascular, si bien esto no es algo nuevo. En muchos países de ingresos bajos se llevan a cabo jornadas internacionales de cirugía cardiovascular pediátrica, cuyo objetivo es mejorar la supervivencia de los pacientes con CC. Pese a ello, los países donde se efectúan son aquellos que, dada la falta de fondos, no cuentan con la estructura médica ni la estructura sanitaria suficiente para estos enfermos; aunque es cierto que se realizan intervenciones y se intenta atender a una proporción de estos individuos, lamentablemente no se mejora el acceso a la mayor parte de la población y en muchos casos no es posible la sostenibilidad de los programas de CC en estos países ${ }^{8}$. En México se observa algo similar con estas fundaciones, ya que son todavía insuficientes ante la gran demanda de pacientes con CC en todo el país a pesar de tales esfuerzos. Además, debe recordarse que el origen de las jornadas de cirugía cardiovascular pediátrica tuvo lugar en países con ingresos sumamente bajos, sin recursos humanos ni materiales suficientes para la atención de las CC. Éste no es el caso de este país: entonces ¿por qué no se ha avanzado en la atención equitativa de las CC?

Las principales barreras para el progreso de la atención de las CC son la falta de financiamiento, el nulo compromiso político y la escasa información de las $\mathrm{CC}^{9}$ :

Falta de financiamiento: Es sin duda uno de los puntos más importantes. La atención médica y quirúrgica de las CC exige muchos recursos. En realidad, a menudo es complejo establecer un diagnóstico preciso y para ello se necesitan modalidades diagnósticas costosas, además de especialistas médicos altamente capacitados. El tratamiento quirúrgico de las CC también consume recursos considerables, infraestructura y equipos complejos, además de profesionales certificados. Obviamente es muy costoso ${ }^{6}$. Esta falta de financiamiento es la principal barrera.

Nulo compromiso político: Muchos políticos entienden la dinámica de las elecciones nacionales y, si quieren reelegirse, necesitan pruebas constatables: establecer y expandir un servicio de cirugía cardiovascular pediátrica pueden consumir un mayor tiempo que el término gubernamental promedio debido a la capacitación de equipos cardiovasculares y la larga curva de aprendizaje para los hospitales 9 ; por lo tanto no es atractiva su aplicación. Si se agrega que el gasto público en salud equivale en México a apenas al $2.5 \%$ del producto interno bruto $(\mathrm{PIB})^{10}$, muy por debajo del $6 \%$ que calcula la Organización para la Cooperación y el Desarrollo Económico (OCDE) para proveer un sistema de salud optimo ${ }^{11}$, entonces se producen grandes limitaciones en el sector salud, incluida la calidad para la atención de los pacientes con CC.

Escasa información de las CC: Se han realizado varios esfuerzos para disponer de datos reales nacionales de las $C C$, pero no se han conseguido a pesar de la importancia y son un impedimento importante porque no pueden obtenerse recursos adecuados para la atención de los pacientes.

Sin lugar a dudas, tanto la falta de financiamiento como el limitado compromiso político son las principales barreras para la atención adecuada de los pacientes con CC en el país; es claro que el problema es multidisciplinario y multisectorial. 
La evolución natural de las CC depende de múltiples factores, entre ellos el tipo de CC, la raza y el tiempo de diagnóstico; uno de los principales es el inicio del tratamiento y por desgracia la localidad geográfica y el estado socioeconómico ${ }^{12}$ son dos de los factores más importantes en México que determinan la calidad y el tipo de atención de los sujetos con CC.

Entre los principales problemas que enfrentan los hospitales públicos para la atención de los pacientes con CC figuran los siguientes:

1. Falta de protocolos de detección de CC prenatal y posnatal, por lo que un número considerable de pacientes con CC críticas se diagnostica cuando está sintomático, en muchas ocasiones luego del cierre del conducto arterioso en los primeros días y semanas de vida, lo que incrementa su morbilidad $y$ mortalidad.

2. Transferencia a hospitales de tercer nivel. Sin duda alguna, durante su traslado, los pacientes con CC que requieren algún tipo de intervención se hallan en estado crítico y en muchas ocasiones requieren desplazamientos a diversos centros hospitalarios, lo cual no sólo conlleva un alto riesgo de mortalidad sino además retraso del tratamiento oportuno.

3. Retraso del tratamiento médico. Es el principal problema e incluso en algunos lugares no puede iniciarse la infusión de prostaglandinas ante un diagnóstico de una CC crítica, un factor esencial para la supervivencia del paciente. Además, en muchos hospitales no es posible efectuar traslados a centros donde está disponible por el alto volumen de pacientes que atienden. En el caso de enfermos que requieren algún tratamiento quirúrgico/intervencionista, en muchos centros hospitalarios del país se pueden practicar operaciones cardiovasculares pediátricas, pero se encuentran limitados por los recursos materiales, con escasos turnos quirúrgicos o un equipo humano incompleto para la atención de las CC. Asimismo, con los cambios en el sistema de salud, en muchos hospitales los familiares deben realizar el pago total de los costos de las intervenciones, algo impensable para muchas familias.

Según el artículo $4^{\circ}$ de la Constitución Mexicana: "Los niños y las niñas tienen derecho a la satisfacción de sus necesidades de alimentación, salud, educación y sano esparcimiento para su desarrollo integral. El Estado otorgará facilidades a los particulares para que se coadyuven al cumplimiento de los derechos de la niñez". La salud es un derecho humano básico. Todos los niños que nacen con CC merecen recibir un tratamiento y tener los accesos a intervenciones capaces de salvar su vida y éstos no deben reservarse para aquéllos nacidos en áreas específicas del país 6 .

\section{Conclusión}

La salud global ha estado dominada por enfermedades contagiosas y la atención primaria materna e infantil ${ }^{9}$. Hasta la fecha, rara vez se aborda la atención de las CC; sin embargo, los hechos no dejan de existir porque se los ignore. Ningún niño con CC debe morir por falta de recursos.

Los servicios de cirugía cardiovascular pediátrica en el país son sumamente limitados para ciertas áreas, en contraste con los grandes avances no sólo en el resto del mundo sino en comparación con los institutos de salud, y la atención de las CC en el país es muy inequitativa. Debido a las posibles implicaciones sociopolíticas, este importante problema de salud no tiene una solución fácil, pese a lo cual es esencial y urgente mejorar la atención cardíaca pediátrica en el país y modificar el sistema de salud, lo cual requiere un enfoque múltiple.

\section{Conflicto de intereses}

Los autores declaran no tener ningún conflicto de intereses.

\section{Responsabilidades éticas}

Protección de personas y animales. Los autores declaran que para esta investigación no se han realizado experimentos en seres humanos ni en animales.

Confidencialidad de los datos. Los autores declaran que en este artículo no aparecen datos de pacientes.

Derecho a la privacidad y consentimiento informado. Los autores declaran que en este artículo no aparecen datos de pacientes.

\section{Bibliografía}

1. Moruno-Tirado A, Santos-de Soto J. Mesa redonda. Avances en especialidades pediátricas. Otras ponencias presentadas en la XCII Reunión científica de la Sociedad de Pediatría de Andalucía Occidental y Extremadura Puerto de Santa María (Cádiz), 10 de Noviembre de 2006. Avances en Cardiología Pediátrica. Vox Paediatrica. 2006; 14(1):56-63.

2. Alva-Espinosa C. Breve perspectiva histórica de las cardiopatías congénitas en el mundo y en México. Evid Med Invest Salud. 2014; 7(S1):S39-43

3. Attie F, Rosas-Peralta M, Pastelín-Hernández G. Pasado, presente y futuro de la cardiología pediátrica. Arch Cardiol Ped. 2006; 76(2):S248-56.

4. Arana-Rueda E, Cabrera-Bueno F, Muñoz-García A, Romero-Rodríguez N, Jiménez-Navarro MF. Cardiología pediátrica: avance continuo. Cardiocore. 2013;48(1):1-2. 
Arch Cardiol Mex. 2020;90(3)

5. Zimmerman MS, Carswell-Smith AG, Sable CA, Echko MM, Wilner LB, Olsen HE, Tasew-Atalay H. Global, regional, and national burden of congenital heart disease, 1990-2017: a systematic analysis for the Global Burden of Disease Study 2017. Lancet Child Adolesc Health 2020 January 21. DOI 10.1016/S2352.4642(19)30402-X

6. Peña-Juárez RA, Medina-Andrade MA. Situación real de las cardiopatías congénitas en dos hospitales públicos del estado de Jalisco. Arch Cardiol Mex. 2019;89(4):1-7.

7. Calderón-Colmenero J, Cervantes-Salazar JL, Curi-Curi PJ, Ramírez-Marroquín S. Problemática de las cardiopatías congénitas en México. Propuesta de regionalización. Arch Cardiol Mex. 2010;80(2):133-140.

8. Nguyenvu N, Leon Wyss J, Krishna SI, Thomas Pezzella A. Paediatric cardiac surgery in low income and middle income countries: a continuing challenge. Arch Dis Chil. 2015;0:1-4
9. Barriers underlying the establishment and expansion of cardiac surgery in low and middle income countries. Global Cardiac Surgery. Disponible en: https://globalcardiacsurgery.com/author/globalcardiacsurgery/. Revisado el 31 de enero 2020.

10. Global Health Expenditure Database. World Health Organization Expediture database. Disponible en: http://apps.who.int/nha/database. Revisado el 31 de enero del 2020.

11. Analisis ex post de la regulacion: prácticas y lecciones de países de la OCDE. OCDE database. Disponible en: http://www.oecd.org/centrodemexico/publicaciones/publicacionesdeladivisiondepoliticaregulatoriadelaocde.htm

12. Ashutosh A, Sethuraman S, Suvarna G, Sudheer G. Border medicine: the pediatric cardiology perspective. Ped Cardiol. 2020;41(1):202-5, doi. org/10.1007/s00246-019-02171-7. 